\title{
A preliminary study on progesterone antioxidation in promoting learning and memory of young ovariectomized mice
}

Lu He, Hui Yang, Li-dong Zhai, Heng Shao, Yun-sheng Li

Department of Anatomy and Neurobiology, Tianjin Medical University, China

Submitted: 8 November 2010

Accepted: 31 January 2011

Ach Med Sci 2011; 7, 3: 397-404

DOI: 10.5114/aoms.2011.23402

Copyright @ 2011 Termedia \& Banach

\section{Abstract}

Introduction: The aim of this preliminary study was to evaluate the effect of long-term progesterone (P4) treatment on structural and functional deficits associated with the hippocampus.

Material and methods: Mice served as sham controls or were bilaterally ovariectomized (Ovx), and a 90-day regimen of placebo or P4 was applied to the animal. After the administration, the acquisition and retrieval of mice in contextual fear conditioning (CFC) and a water maze were examined. Hippocampal tissues from some mice in each group were stained with cresyl violet, and the remainder were taken for determining the antioxidant power. Results: Compared with placebo controls, the time spent on freezing was higher and the latencies were longer for mice given high-dose P4 (HP) $(p<0.05)$ in CFC, and the HP group also had longer searching time spent in the target quadrant $(p<0.05)$ in the water maze. Compared with placebo controls, the cell number of hippocampus CA1, CA3 and DG was significantly higher in the HP group $(p<0.05)$, and the thickness of the cell layer in CA1 and DG was also higher in the HP group $(p<0.05)$. All the oxidative stress biomarkers show that the antioxidative activity in hippocampus tissue from the HP and LP groups is higher than that in placebo controls $(p<0.05)$.

Conclusions: Ovx impairs learning and memory of mice, which can be rescued by a long-term regimen of HP via its antioxidant effects.

Key words: progesterone, contextual fear conditioning, water maze, Nissl stain, hippocampus, antioxidation.

\section{Introduction}

Progesterone (P4) has been reported to be neuroprotective against oxidative stress, excitatory neurotoxicity, ischaemia and post-injury cerebral oedema [1-3]. Some studies suggest that, like 17 $\beta$-oestradiol (E2), P4 can enhance memory. For example, a single injection of P4 given immediately after training enhances object recognition, working memory, and inhibitory avoidance in young ovariectomized (Ovx) rats [4]. Indeed, an acute physiological dose with $\mathrm{P} 4$ would enhance cognitive performance of Ovx mice in several cognitive measures, such as spontaneous alternation and object recognition tasks [5-9]. Variations in cognitive performance of female rodents over reproductive cycles coincide with changes in levels of ovarian hormones. Changes in endogenous hormones influence cognitive performance, and pregnant rats' performance in the water maze task is enhanced when E2 and

\author{
Corresponding author: \\ Yun-sheng Li \\ Department of Anatomy \\ and Neurobiology \\ Tianjin Medical University \\ Tianjin 300070, China \\ E-mail: liyunsh@126.com
}


P4 levels are escalating in the $1^{\text {st }}$ and $2^{\text {nd }}$ trimester compared with non-pregnant rats [5]. Many of the previous studies show that P4 alone can enhance cognitive performance of aged male and female mice in hippocampal tasks, and the cognitive effects of P4 involve pre/post-training administration and/or testing when hormone levels were still high under a shortterm regime [6-8]. Thus, P4 may have actions on the hippocampus to improve learning and memory of mice. Moreover, new evidence has been found that $\mathrm{P} 4$ produces beneficial effects on cognitive performance of mice with a long-term treatment [9]. Even so, further studies should assess how this important ovarian hormone modulates cognitive function.

There exists age-dependent endogenous protection in brain tissue which induces activation and/or synthesis of proteins and genes, since the intermediate age subgroup is more tolerant to hypoxic-ischaemic brain damage than either very young or more mature ages [10]. Hormone deficiency during aging may render the hippocampus more vulnerable to deterioration and exacerbate memory deficits [11]. P4 significantly alters hippocampal physiology by modulating CA1 dendritic spine density, synaptic proteins, and intracellular signalling $[12,13]$. P4 also seems to be beneficial in preventing mitochondrial dysfunction that results in loss of hippocampal cells after a controlled cortical contusion $[14,15]$. Female rats given P4 supplements via silastic implants had no loss of mitochondrial oxygen consumption and there was virtually complete preservation of hippocampal neurons in the CA1 and CA3 subfields of the hippocampus [2]. That P4 can alone exert chronic trophism on the hippocampus may be connected with certain trophic factors, including that P4 reduces lipid peroxidation and oxidative stress by decreasing the generation of free radicals and enhancing endogenous free-radicalscavenging systems $[2,16]$. As such, the effects of P4 on the hippocampus in young females may be highly dependent on its antioxidation.

Of interest is whether $\mathrm{P} 4$ protects the hippocampus by reducing lipid peroxidation and oxidative stress to improve learning and memory of female mice. We evaluated the effect of long-term treatment with P4 on young Ovx mice in hippocampus measures. Then, we examined the histology and oxidative stress biomarkers in the hippocampus of the subjects to test our hypothesis that $\mathrm{P} 4$ has protective actions via antioxidation on the hippocampus.

\section{Material and methods}

\section{Animals and drugs}

Six-week-old female Kunming mice (22-25 g) were assigned randomly to a sham operated control (Sham) group, low-dose P4 (LP) group, high-dose
P4 (HP) group and placebo control group. Mice had the ovaries excised under diethyl ether anaesthesia except the sham group, in which only the fat around the ovaries was removed. All procedures were approved by Tianjin Medical University Animal Care Committee.

P4, purchased from Xianju Pharmacy (China), was given to the mice in LP $(10 \mathrm{mg} / \mathrm{kg})$ and HP (30 mg/kg) once per 5 days by sc injection and lasted 90 days. The $10 \mathrm{mg} / \mathrm{kg}$ dose had been used previously in adult Ovx mice and this dose was estimated in the physiological range $[5,7,8,17]$. The $30 \mathrm{mg} / \mathrm{kg}$ dose was used in case young females were insensitive to physiological doses. Mice in sham and placebo groups were treated with physiological saline in the same way. All administration was started after complete recovery from the ovariectomy (day 7).

\section{Behavioural testing}

The primary measures for each task were performed after finishing the administration of P4, which is described below.

Contextual fear conditioning (CFC): the mice were trained and tested in a modified CFC task developed by LaLumiere [18]. The CFC was assessed by placing each mouse in a Y-maze composed of three equally spaced arms. One arm was designated as the shock alley, and the other two arms were non-shock alleys. The task lasted 3 days. On day 1, each mouse was placed into one of the three arms and allowed to explore the entire maze for $8 \mathrm{~min}$. On day 2, the shock arm was blocked off from the rest of the maze by an opaque door. Each mouse was placed in the shock arm and 1 min later received 2 footshocks $(2 \mathrm{~s}$, $0.5 \mathrm{~mA}, \mathrm{AC})$, the first after a delay of $15 \mathrm{~s}$ and the second after another delay of $15 \mathrm{~s}$. On day 3 , each mouse was placed into a non-shock alley and permitted to explore the entire maze freely for $8 \mathrm{~min}$. Footshock was not administered during the test. The freezing time and the latency of first entering the shock alley were used as indices of retention of the footshock training. Freezing was defined as the cessation of all movement except what was necessary for respiration.

Water maze task: the Morris water maze test was performed according to previously described methods [19]. Mice were habituated to the maze by allowing them to swim in the water maze with the hidden platform in it. After 2 min, mice were placed on the hidden platform for $10 \mathrm{~s}$. Following habituation, mice were trained for 9 days with four trials per day at inter-trial intervals of 40-50 min. Performance in the spatial learning task was measured by timing escape latencies during each training session. The escape latencies of four trials per day were averaged for each animal. To assess retention, mice were tested in a 1 min probe trial 1 day later, in which the platform 
was not available for escape. The duration spent in the target quadrant where the platform was located during training was recorded.

\section{Histological examination}

At the completion of all behavioural testing, mice were sacrificed with cervical dislocation. The brains of six mice in each group were embedded in paraffin, cut into $8-\mu m$-thick coronary sections, and stained with cresyl violet. Photographs of the hippocampus of each specimen were taken by using Olympus biological microscope CX31. The cellular integrity and thickness of the pyramidal layer and granular layer in regions CA1 and CA3 and the dentate gyrus (DG) of the hippocampus were measured. High Definition Medical Image Analysis Program was used for counting the cell number $(\times 1000)$ and the thickness $(\times 400)$ of the cell layer performed on three random fields in each section. Cells which had a shrunken body with surrounding empty spaces were excluded.

\section{Assay of oxidative stress biomarkers}

The brains of the remaining mice in each group were quickly removed followed by careful dissection of the hippocampus in ice-cold saline. Homogenate (10\%) was made from the hippocampus tissue after cutting it into pieces under cold conditions. Protein content was first detected based on the Bradford method, then the levels of superoxide dismutase (SOD), malic dialdehyde (MDA) and total antioxidant capability (T-AOC) in tissue were measured by using detection kits which were purchased from Jiancheng Bioengineering (China).

\section{Statistical analysis}

The behavioural and biochemical effects were analysed by one-way ANOVA using SPSS 15 soft- ware. Least-significant difference (LSD) comparisons were used to ascertain groups that were different. The result data are presented as mean \pm SEM (standard error of mean). The $p<0.05$ level of probability was used as the criterion of significance.

\section{Results}

\section{Contextual fear conditioning}

A difference was found in the duration of freezing among the groups (Figure $1 \mathrm{~A}$ ). The time spent on freezing was higher for the sham and HP groups compared with placebo control animals $(p<0.05)$. The amount of time spent on freezing during the test was not compared with the freezing time during the habituation because none of the animals froze during habituation. A difference was found in the entrance latencies among the groups (Figure $1 \mathrm{~B}$ ). The latencies of the sham and HP groups were longer than those of the placebo group $(p<0.05)$.

\section{Water maze testing}

The training latencies descended sharply during the first 5 training days, and there were differences for sham and HP groups versus the placebo group except for training days 1,2 and 4 ( $p<0.05$, Figure $2 \mathrm{~A}$ ). In the probe trial, sham and HP groups had a longer searching time spent in the target quadrant than mice treated with placebo $(p<0.05$, Figure 2 B).

\section{Histological examination}

Nissl stain further revealed a mismatch between the placebo and HP treatments (Figure 3). Compared with the placebo group, the cell numbers of the CA1, CA3 and DG are all higher in the sham and HP groups ( $p<0.05$, Figure 4 A). Compared with the placebo group, the thicknesses of the
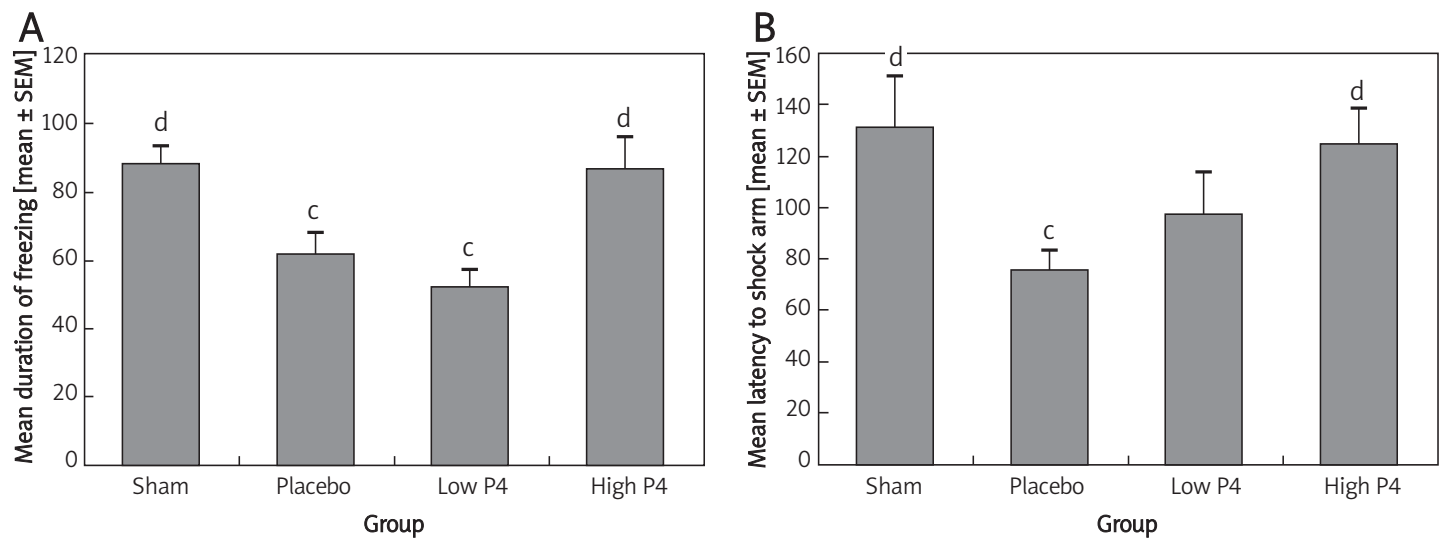

Figure 1. Results of CFC task. (A) Time spent on freezing on the retention test day. (B) Shock arm entrance latencies during the test

Data are means \pm SEM, $n=12$ for sham and $L P, n=11$ for placebo and $H P,{ }^{c} p<0.05$ vs. sham group, ${ }^{d} p<0.05$ vs. placebo group 
A

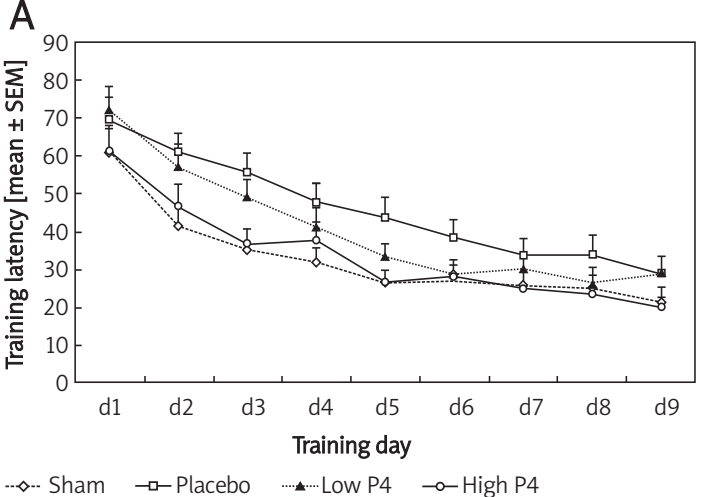

B

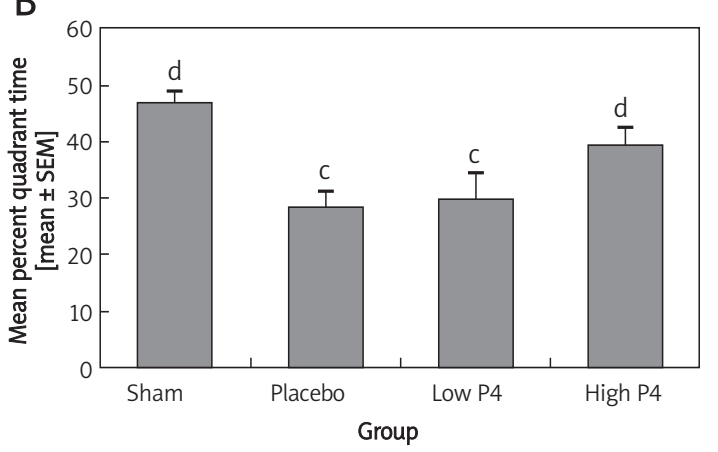

Figure 2. Results of water maze task. (A) Latency during training stage. (B) Percent quadrant time during probe test. Data are means $\pm S E M, n=12$ for sham and $L P, n=11$ for placebo and HP, ${ }^{c} p<0.05$ vs. sham group, ${ }^{d} p<0.05$ vs. placebo group

pyramidal layer of CA1 and the granular layer of DG are both higher in the sham and HP groups, and the thickness of the pyramidal layer of CA3 is also higher in the sham group ( $p<0.05$, Figure 4B).

\section{Assay of antioxidative activity}

Compared with the placebo group, SOD levels of hippocampus tissue are higher in the LP, HP and sham groups ( $p<0.05)$, MDA levels of hippocampus tissue are lower in the LP, HP and sham groups, and the T-AOC of hippocampus tissue was higher in the HP and sham groups $(p<0.05)$ (Table I).

\section{Discussion}

The behavioural tests in the present experiment prove that a long-term regime of $\mathrm{P} 4$ improves the learning and memory of young Ovx mice in CFC and the water maze task, independently of E2. These findings are in agreement with extensive evidence that P4 enhances hippocampal-dependent memory [5-9]. In addition to this, we found that LP did not affect the subsequent retention performance in CFC training and the water maze task. So the findings provide additional evidence that the memoryenhancing effects of P4 are dose dependant. Considerable evidence indicates that the hippocampus is involved in memory for the CFC task, which requires learning of contextual cues [20]. Because the mice were placed and retained in the compartment where a shock was delivered during the CFC training, the mice had an opportunity for learning any escape or avoidance response. Also, the mice's latency to enter the shock compartment was used to assess retention of the training [21]. Mice given HP had entrance latencies that were longer than those of the placebo controls in the CFC task. Freezing behaviour in the retention test is clearly required for the evidence of mice's retention of CFC training. The mice given HP displayed significantly more freezing in the retention test compared with that of the placebo controls. Since P4 acts at the GABA receptor through its metabolites, such as allopregnanolone, which is a positive allosteric modulation of the GABA type A (GABA-A) receptor [15], it is interesting that animals treated with $\mathrm{P} 4$ show more freezing after the abrupt withdrawal of such a large and consistent dose of P4. Here, two aspects may be considered to cause this type of behaviour. First, P4 and its metabolites may be retained in the plasma membrane and/or in the intracellular compartments, constituting a reserve modulating the GABA-A system for a long time after the initial exposure [22]. They are also accumulated in the intracellular compartment and then re-supply the plasma membrane with steroids able to modulate the GABA-A receptor, so that the rate of this transport can affect the kinetics of P4 actions [23]. Secondly, P4 and its metabolites regulate both the expression and the function of GABA-A receptor subunits [24]. In hippocampal CA1 of female rats, 2-day exposure to pregnenolone or E2 plus P4 increased the expression of the GABA-A $\delta$ subunit [25].

In the water maze, mice are trained to navigate to a platform located below the water surface. Spatial learning is then typically assessed in a probe test, where the platform is removed from the pool and the mouse is allowed to search for it. Performance in the probe test is evaluated using the percent time in a virtual quadrant or zone centred on the former platform location [26]. The data from the water maze task demonstrated that the escape latency descended rapidly in the first five days and became steady five days later. During the period when latency descended quickly, HP had a notable effect on improving the performance of the Ovx mice on the third and fifth day. The preliminary study indicated that long-term lack of ovarian hormone would damage the spatial cognition of mice in the water maze task, and the latencies to find the platform show significant 


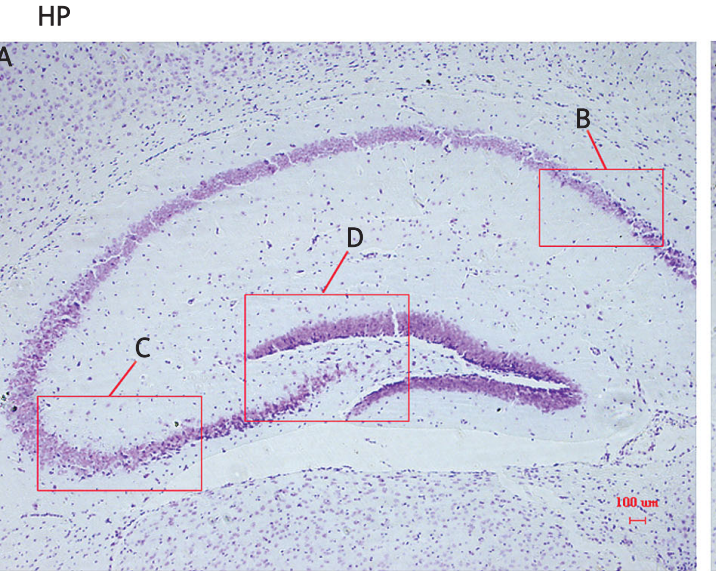

\section{Placebo}
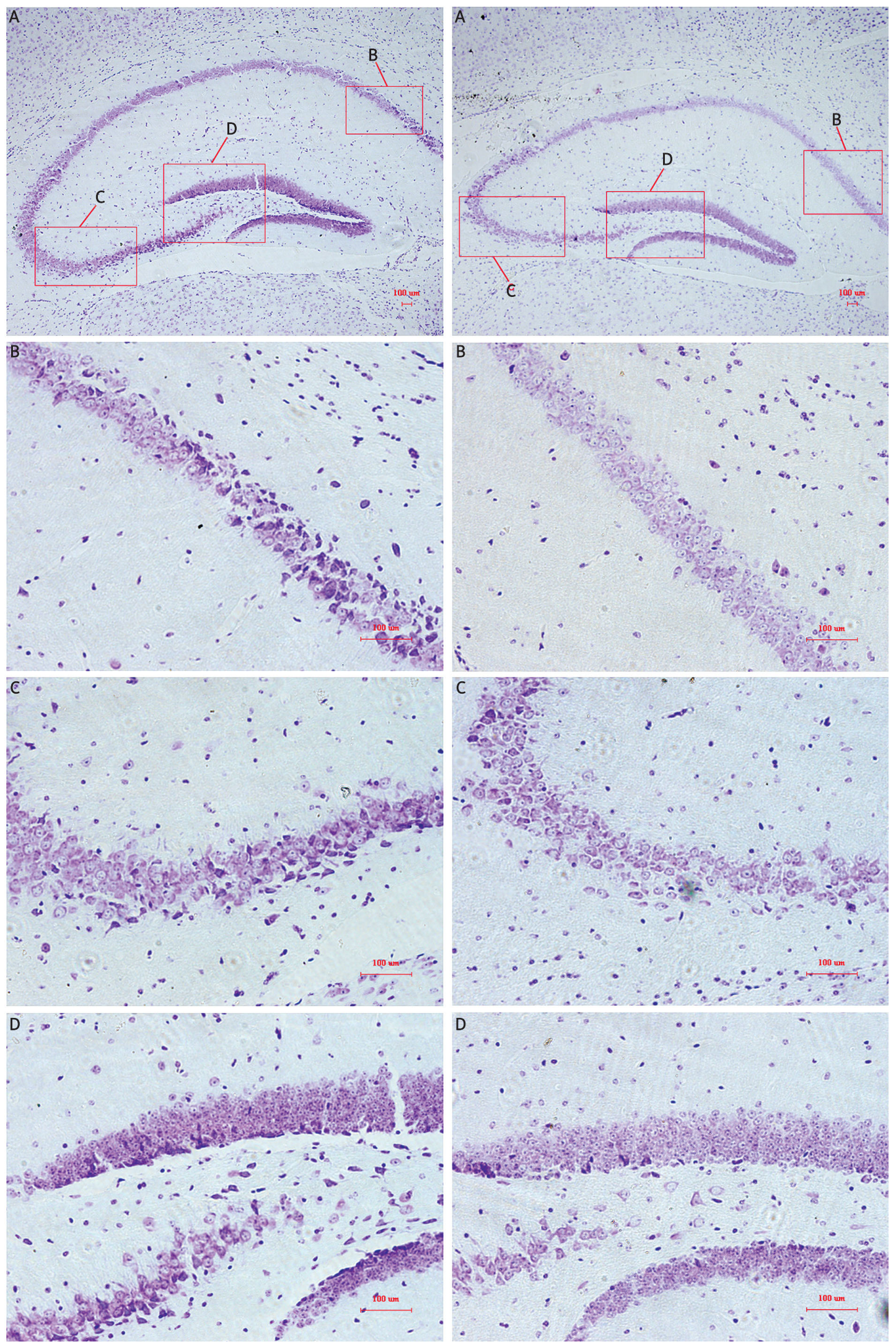

Figure 3. Coronary section of left hippocampus from HP (left) and placebo (right) groups. B, C and D $(\times 200)$ show the magnification of the square areas in A $(\times 40)$. (B) sub-region CA1. (C) sub-region CA3. (D) sub-region DG. Scale bars $100 \mu \mathrm{m}$ 

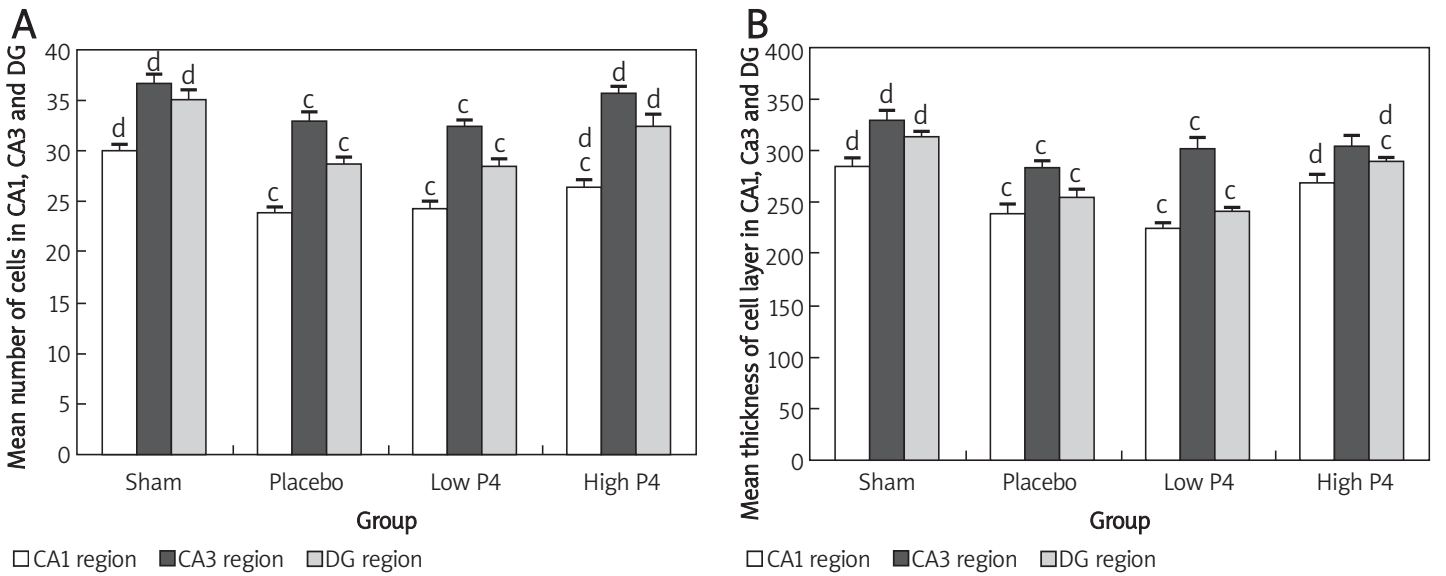

Figure 4. Examination results of the histological section of hippocampus. (A) Number of Nissl-stained cells (cells/cm², oil) in sub-regions CA1, CA3 and DG. (B) Thickness of pyramidal layer in sub-regions CA1 and CA3, and thickness of granular layer in sub-region DG

Data are means \pm SEM, $n=6$ for each group, ${ }^{c} p<0.05$ vs. sham group, ${ }^{d} p<0.05$ vs. placebo group

Table I. Means ( \pm SEM) of the levels of malic dialdehyde (MDA), superoxide dismutase (SOD) and the activity of total antioxidant capability (T-AOC)

\begin{tabular}{|llccc|}
\hline Group & $n$ & MDA [nmol/mg] & SOD [NU/mg] & T-AOC [U/mg] \\
\hline Sham & 6 & $4.344 \pm 0.581^{\mathrm{d}}$ & $181.752 \pm 6.059^{\mathrm{d}}$ & $1.824 \pm 0.339^{\mathrm{d}}$ \\
\hline Placebo & 5 & $6.833 \pm 0.955^{\mathrm{c}}$ & $153.952 \pm 6.845^{\mathrm{c}}$ & $0.927^{\mathrm{c}} \pm 0.273^{\mathrm{c}}$ \\
\hline LP & 6 & $5.782 \pm 0.451^{\mathrm{c}, \mathrm{d}}$ & $172.516 \pm 7.567^{\mathrm{c}, \mathrm{d}}$ & $1.316 \pm 0.273^{\mathrm{c}, \mathrm{d}}$ \\
\hline HP & 5 & $4.386 \pm 0.605^{\mathrm{d}}$ & $173.715 \pm 8.196^{\mathrm{d}}$ & $1.759 \pm 0.262^{\mathrm{d}}$ \\
\hline
\end{tabular}

$c_{p}<0.05$ vs. sham group, $d_{p}<0.05$ vs. placebo group

shortening in the Ovx mice with long-lasting application of P4. The results reveal that P4 exerts a beneficial effect during the early stage of learning, and little effect on the conformation of memory. In support, mice that received P4 spent more time in the platform quadrant than those in the Ovx group. Thus, the present findings indicate that long-term P4 treatment influences the consolidation of learning and memory for Pavlovian training experiences, which is consistent with those of other experiments using CFC training as well as water maze training [9].

As CFC and the water maze task involve the hippocampus, these data suggest a role of the hippocampus as a target for P4's effects for cognitive processes. We chose cresyl violet to stain the brain slice, since all cells contain Nissl bodies (rough endoplasmic reticulum and polyribosomes) which can be stained by cresyl violet. The volume and the amount of Nissl bodies reflect the function of protein synthesis in nerve cells. Neural activity, with a large quantity of Nissl bodies, is stronger than that in the damaged nerve cells, in which Nissl bodies shrink or even disappear. So the difference in shade of staining shows that the volume and the amount of Nissl bodies vary among the groups. The CA1, CA3 and DG of the hippocampus were investigated in the present study, and we found that P4 prevents hippocampus cell loss induced by a sharp decline of ovarian hormones. So P4 is shown to promote neuronal cell survival and to have a neuroprotective effect on the hippocampus of the Ovx mice. Here we show that the mice with P4 treatment have a higher cell density compared with the placebo treatment group, and these findings are consistent with the results of the behavioural testing in the present experiment that the HP group has a better learning and memory capacity. This improved performance could be due to reduced neuronal cell death in the CA1, CA3 and DG sub-region. This phenomenon is also observable in other studies: exogenous P4 can protect hippocampal hilar neurons from excitotoxicity degeneration, and the timing of exposure to ovarian hormones may influence the vulnerability of hilar neurons to excitotoxicity $[6,27]$. Although not investigated in an excitotoxicity degeneration study, our study suggests that P4 administration is required to achieve cognitive improvement and reduce neuron death, and that the protection of $\mathrm{P} 4$ differs under different dose conditions.

Malic dialdehyde is one of the most frequently used indicators of lipid peroxidation. Reactive oxygen species degrade polyunsaturated lipids, forming MDA that is used as a biomarker to measure the level of oxidative stress in an organism 
[28]. Superoxide dismutase is a cytosolic enzyme that converts superoxide radicals to hydrogen peroxide, thus protecting the cell from superoxide toxicity [29]. As such, it is an important antioxidant defence in nearly all cells exposed to oxygen. Total antioxidant capability is a biomarker for oxidative stress in tissue, and low T-AOC could be indicative of oxidative stress or increased susceptibility to oxidative damage. Previous data offer evidence that gonadal steroids exert a remarkable neuroprotective effect on reducing oxidative stress in transient global cerebral ischaemia. For instance, P4 appeared to show a regional effect on lipid peroxidation and SOD homeostasis in rat brain, and MDA levels of hippocampus and striatum decreased significantly in P4-treated animals [30]. The results of antioxidative activity in mice hippocampus display a higher SOD level and a lower MDA level in LP, HP and sham groups, which suggests that both low and high doses of P4 have an antioxidative effect. However, these findings are not consistent with the results of the behavioural testing in the present experiment that only the HP group has a better learning and memory capacity. The inharmonious results suggest that two different mechanisms exist in the regulation of P4's action on cell survival and oxidative stress. P4 can be reduced to $5 \alpha$-dihydroprogesterone, which can be further converted to allopregnanolone in the brain [15]. Allopregnanolone has been demonstrated to reduce the levels of reactive oxygen species and lipid peroxidation and prevent peroxide-induced apoptosis and NF- $\kappa \mathrm{B}$ activation, due to its ability to restore the intracellular redox state [31]. In contrast to antioxidation, there are few reports about the ameliorating effects of allopregnanolone on memory. So P4 and its metabolites can only show better improvement in antioxidation, and have little effect on learning and memory. Although investigated in a handful of studies, acute or chronic treatment with P4 in a cerebral ischaemia model has been shown to attenuate brain lipid peroxidation [2, 32]. We observed in the hippocampus that P4 was not as effective in reducing lipid peroxidation as endogenous gonadal hormones in the sham group. These findings appear to be in accord with a non-genomic process and might be viewed as evidence of antioxidant actions of combined treatments with both E2 and P4, as some investigators have suggested [33].

Evidence in the literature generally supports the notion that there is increased damage following ischaemia in aged animals whose gonadal hormones are low [10]. As to females, Ovx may render the neurons more vulnerable to deterioration and exacerbate age-related memory deficits. Oxidative damage to mitochondria is posited to play a major role in aging populations associated with cognitive decline [34]. The results of the present histological survey show that HP can promote neuronal cell survival in the hippocampus of the Ovx mice, which is in cooperation with its excellent antioxidative effect. These findings are also consistent with the behavioural testing showing that the HP group has a better learning and memory capacity. Together, the present data suggest that P4 might be beneficial in the treatment of the cognitive deficit caused by Ovx, at least in part, due to its protective effect on the hippocampus against overoxidation-induced cell death. Moreover, P4 facilitates the learning and memory of Ovx mice, which differs under different dose conditions. As to antioxidation, both high and low levels of P4 are effective in reducing free radical damage.

In conclusion, this preliminary study demonstrates that long-lasting application of $\mathrm{P} 4$ reduces the damage to the learning and memory caused by the lack of ovarian hormone in Ovx mice. Furthermore, P4 exerts a protective effect on the hippocampus against overoxidation-induced cell death, which plays a key role in improving the capacity of the cognitive performance in Ovx mice. Nevertheless, further studies are required to delineate more clearly the signal transduction pathway in the antioxidative effect of P4 on the hippocampus, and the beneficial effects on learning and memory are worth researching further in a clinical trial.

\section{Acknowledgments}

This study was supported by Tianjin Medical University key research project fund (No. 052200010). We thank Professor Xin TIAN for her invaluable assistance with the behavioural processes. The work was conducted at Tianjin Medical University.

\section{References}

1. De Castilhos J, Hermel EE, Rasia-Filho AA, Achaval M. Influence of substitutive ovarian steroids in the nuclear and cell body volumes of neurons in the posterodorsal medial amygdala of adult ovariectomized female rats. Neurosci Lett 2010; 469: 19-23.

2. Stein DG. Progesterone exerts neuroprotective effects after brain injury. Brain Res Rev 2008; 57: 386-97.

3. Schumacher M, Guennoun R, Ghoumari A, et al. Novel perspectives for progesterone in hormone replacement therapy, with special reference to the nervous system. Endocr Rev 2007; 28: 387-439.

4. Walf AA, Rhodes ME, Frye CA. Ovarian steroids enhance object recognition in naturally cycling and ovariectomized, hormone-primed rats. Neurobiol Learn Mem 2006; 86: 35-46.

5. Galea LA, Ormerod BK, Sampath S, Kostaras X, Wilkie DM, Phelps MT. Spatial working memory and hippocampal size across pregnancy in rats. Horm Behav 2000; 37: 86-95. 
6. Frye CA, Walf AA. Progesterone to ovariectomized mice enhances cognitive performance in the spontaneous alternation, object recognition, but not placement, water maze, and contextual and cued conditioned fear tasks. Neurobiol Learn Mem 2008; 90: 171-77.

7. Frye CA, Walf AA. Progesterone enhances performance of aged mice in cortical or hippocampal tasks. Neurosci Lett 2008; 437: 116-20.

8. Lewis MC, Orr PT, Frick KM. Differential effects of acute progesterone administration on spatial and object memory in middle-aged and aged female C57BL/ 6 mice. Horm Behav 2008; 54: 455-62.

9. Frye CA, Walf AA. Effects of progesterone administration and APPswe+PSEN1Deltae9 mutation for cognitive performance of mid-aged mice. Neurobiol Learn Mem 2008; 89: 17-26

10. Schaller BJ. Influence of age on stroke and preconditioning-induced ischemic tolerance in the brain. Exp Neurol 2007; 205: 9-19.

11. Driscoll I, Sutherland RJ. The aging hippocampus: navigating between rat and human experiments. Rev Neurosci 2005; 16: 87-121.

12. Choi JM, Romeo RD, Brake WG, Bethea CL, Rosenwaks Z, McEwen BS. Estradiol increases pre- and post-synaptic proteins in the CA1 region of the hippocampus in female rhesus macaques (Macacamulatta). Endocrinology 2003; 144: 4734-8.

13. Nilsen J, Brinton RD. Divergent impact of progesterone and medroxyprogesterone acetate (Provera) on nuclear mitogen-activated protein kinase signaling. Proc Natl Acad Sci USA 2003; 100: 10506-11.

14. Robertson CL, Puskar A, Hoffman GE, Murphy AZ, Saraswati M, Fiskum G. Physiologic progesterone reduces mitochondrial dysfunction and hippocampal cell loss after traumatic brain injury in female rats. Exp Neurol 2006; 197: 235-43.

15. Schumacher M, Akwa Y, Guennoun R, et al. Steroid synthesis and metabolism in the nervous system: trophic and protective effects. J Neurocytol 2000; 29: 307-26.

16. Irwin RW, Yao J, Hamilton RT, Cadenas E, Brinton RD, Nilsen J. Progesterone and estrogen regulate oxidative metabolism in brain mitochondria. Endocrinology 2008; 149: $3167-75$.

17. Harburger LL, Bennett JC, Frick KM. Effects of estrogen and progesterone on spatial memory consolidation in aged females. Neurobiol Aging 2007; 28: 602-10.

18. LaLumiere RT, Buen TV, McGaugh JL. Post-training intrabasolateral amygdala infusions of norepinephrine enhance consolidation of memory for contextual fear conditioning. J Neurosci 2003; 23: 6754-8.

19. Dai JX, Han HL, Tian M, et al. Enhanced contextual fear memory in central serotonin-deficient mice. Proc Natl Acad Sci USA 2008; 105: 11981-6.

20. Buckley MJ. The role of the perirhinal cortex and hippocampus in learning, memory, and perception. Q J Exp Psychol B 2005; 58: 246-68.

21. Petrulis A, Eichenbaum $\mathrm{H}$. The perirhinal-entorhinal cortex, but not the hippocampus, is critical for expression of individual recognition in the context of the Coolidge effect. Neuroscience 2003; 122: 599-607.

22. Belelli D, Herd MB. The contraceptive agent Provera enhances GABA-A receptor-mediated inhibitory neurotransmission in the rat hippocampus: evidence for endogenous neurosteroids? J Neurosci 2003; 23: 10013-20.

23. Akk G, Shu HJ, Wang C, et al. Neurosteroid access to the GABA-A receptor. J Neurosci 2005; 25: 11605-13.
24. Follesa P, Biggio F, Caria S, Gorini G, Biggio G. Modulation of GABA-A receptor gene expression by allopregnanolone and ethanol. Eur J Pharmacol 2004; 500: 413-25.

25. Shen H, Gong QH, Yuan M, Smith SS. Short-term steroid treatment increases delta GABA-A receptor subunit expression in rat CA1 hippocampus: pharmacological and behavioral effects. Neuropharmacology 2005; 49: 573-86.

26. Morris RG, Garrud P, Rawlins JN, O'Keefe J. Place navigation impaired in rats with hippocampal lesions. Nature 1982; 297: 681-3.

27. Moralí G, Letechipía-Vallejo G, López-Loeza E, Montes P, Hernández-Morales L, Cervantes M. Post-ischemic administration of progesterone in rats exerts neuroprotective effects on the hippocampus. Neurosci Lett 2005; 382: 286-90.

28. Del Rio D, Stewart AJ, Pellegrini N. A review of recent studies on malondialdehyde as toxic molecule and biological marker of oxidative stress. Nutr Metab Cardiovasc Dis 2005; 15: 316-28.

29. Petkau A. Role of superoxide dismutase in modification of radiation injury. Br J Cancer Suppl 1987; 8: 87-95.

30. Ozacmak VH, Sayan H. The effects of 17 beta estradiol, 17alpha estradiol and progesterone on oxidative stress biomarkers in ovariectomized female rat brain subjected to global cerebral ischemia. Physiol Res 2009; 58: 909-12.

31. Zampieri S, Mellon SH, Butters TD, et al. Oxidative stress in NPC1 deficient cells: protective effect of allopregnanolone. J Cell Mol Med 2009; 13: 3786-96.

32. Cai W, Zhu Y, Furuya K, Li Z, Sokabe M, Chen L. Two different molecular mechanisms underlying progesterone neuroprotection against ischemic brain damage. Neuropharmacology 2008; 55: 127-38.

33. Aggarwal R, Medhi B, Pathak A, Dhawan V, Chakrabarti A. Neuroprotective effect of progesterone on acute phase changes induced by partial global cerebral ischaemia in mice. J Pharm Pharmacol 2008; 60: 731-7.

34. Serrano F, Klann E. Reactive oxygen species and synaptic plasticity in the aging hippocampus. Ageing Res Rev 2004; 3: 431-43. 